\title{
Prediction was predictable from human brain activity in fronto-parietal cortex
}

\author{
Yumi Shikauchi ${ }^{1,2^{*}}$, Ishii Shin ${ }^{1,2}$ \\ From Twenty Second Annual Computational Neuroscience Meeting: CNS*2013 \\ Paris, France. 13-18 July 2013
}

In partially observable environments, prediction of the future is a key to make appropriate decisions [1,2]. Whether or not to open the door (decision making) would depend on whether one could expect someone is behind the door (prediction) even in a well-known environment such as an office. Such interpolation of pre-observed information was obtained by integrating the past observations and the environment model. Recent evidence suggests that such model-based decision-making activates fronto-parietal cortex [3-6]. Despite popularity of neural decoding, research on mapping from neural signals into prediction of pre-observed information has rarely been reported.

Here, we show that prediction decoding is possible from individuals' functional magnetic resonance imaging (fMRI) activity. We asked four healthy subjects to perform a scene prediction task, which required discriminating a true next scene in a three-dimensional navigation environment. We decoded the predicted scene consisting of three views; forward-center (fC), forward-left (fL) and forwardright (fR), information from fMRI activity in anatomically defined region of interests (ROIs): lateral prefrontal cortex (LPF), medial prefrontal cortex (MPF) and parietal cortex (PC). The decoding analysis was conducted individually for each subject and ROIs and the performance values across subjects were then averaged.

All subjects carried out the scene prediction task $92.6 \% \pm 3.2 \%$ correct and $4.8 \% \pm 3.5 \%$ incorrect. Using a leave-one-trial-out procedure, we decoded individuals predicted scene per view from the fMRI data of correct trials. When using PC activity, these decoders allowed us to read out predicted all views (fC: $70.94 \%$; $\mathrm{p}<0.01 \times 10^{-1}$, fL: $60.43 \% ; \mathrm{p}<0.01$, fR: 65.18\%; $\mathrm{p}<0.05)$. In contrast,

\footnotetext{
* Correspondence: yumi-s@sys.i.kyoto-u.ac.jp

'Graduate School of Informatics, Kyoto University, Gokasho, Uji, Kyoto 6110011, Japan

Full list of author information is available at the end of the article
}

above-chance performance was obtained fC view only in LPF and MPF (LPF: 67.78\%; p < $0.01 \times 10^{-3}$, MPF: 62.97\%; $\mathrm{p}<0.05)$. Remarkably, PC bit decoders that trained by correct trials can also read out incorrect scene selected by the subject (fC: $66.77 \%$; $<$ < 0.01, fL: 58.19\%; $\mathrm{p}=0.43$, fR: $75.43 \%$; $\left.<0.05 \times 10^{-1}\right)$.

In this study, we demonstrated decoding of scene prediction from individuals fMRI activity. Because $\mathrm{fC}$ is the most important view for the subjects to determine the next motion, the $\mathrm{fC}$ decoders show the high decoding accuracy for all ROIs. These results suggest that prediction could be performed in the fronto-parietal network such to reflect the degree of contribution to the subsequent decision-making. Our findings have outlined the decision making system employed in complicated environments, and implied useful characters of decoders which can be used for brain machine interface of practical navigation systems.

\section{Acknowledgements}

This research was supported by JSPS KAKENHI Grant Number 715121400004 and a contract with the Ministry of Internal Affairs and Communications entitled, 'Novel and innovative R\&D making use of brain structures'.

\section{Author details}

'Graduate School of Informatics, Kyoto University, Gokasho, Uji, Kyoto 6110011, Japan. ${ }^{2}$ ATR Neural Information Analysis Laboratories, 2-2-2 Hikaridai Seika-cho, Sorakugun, Kyoto, 619-0288 Japan.

Published: 8 July 2013

\section{References}

1. Sutton RS, Barto AG: Reinforcement Learning: An Introduction. MIT Press; 1998.

2. Kaelbling LP, Littman ML, Cassandra AR: Planning and acting in partially observable stochastic domains. Artif Intell 1998, 101(1-2):99-134.

3. Daw N, Gershman SJ, Seymour B, Dayan P, Dolan RJ: Model-based influences on humans' choices and striatal prediction errors. Neuron 2011, 69(6):; 1204-1215.

4. Gläscher J, Daw N, Dayan P, O'Doherty JP: States versus rewards: dissociable neural prediction error signals underlying model-based and model-free reinforcement learning. Neuron 2010, 66(4):585-595.

\section{Biomed Central}


5. Yoshida $\mathrm{W}$, Ishii S: Resolution of uncertainty in prefrontal cortex. Neuron 2006, 50(5):781-789.

6. Bollinger J, Rubens MT, Zanto TP, Gazzaley A: Expectation-driven changes in cortical functional connectivity influence working memory and longterm memory performance. J Neurosci 2010, 30(43):14399-14410.

doi:10.1186/1471-2202-14-S1-P181

Cite this article as: Shikauchi and Shin: Prediction was predictable from human brain activity in fronto-parietal cortex. BMC Neuroscience 201314 (Suppl 1):P181.

Submit your next manuscript to BioMed Central and take full advantage of:

- Convenient online submission

- Thorough peer review

- No space constraints or color figure charges

- Immediate publication on acceptance

- Inclusion in PubMed, CAS, Scopus and Google Scholar

- Research which is freely available for redistribution 